\title{
Cientimates, un recurso lúdico para la enseñanza de las ciencias y las matemáticas en Educación Infantil
}

\author{
Nerina Muela Avilés, Alicia Fernández-Oliveras y María Luisa Oliveras. Universidad de \\ Granada \\ Recepción: 12 de abril de 2015 | Revisión: 30 de mayo de 2015 | Aceptación/Publicación: 24 de julio de 2015 \\ Correspondencia: nerema@correo.ugr.es | alilia@ugr.es | oliveras@ugr.es | http://hdl.handle.net/10481/37120
}

\begin{abstract}
Resumen: Con el trabajo resumido en este artículo, se ha realizado el estudio, diseño y desarrollo de un juego educativo dirigido a niños de Educación Infantil, como herramienta que ayude a mejorar la comprensión de algunos conceptos relacionados con las ciencias y las matemáticas en esta etapa. Entre los autores que constituyen la fundamentación teórica del recurso que hemos elaborado se encuentra Rousseau (1985), partidario del aprendizaje por experimentación, Froebel (1902), precursor de la actividad lúdica para aprender, Decroly (1965), que apoyaba el valor didáctico de materiales de uso común en la vida cotidiana y juegos fabricados en casa, y, especialmente, Montessori (1937), por tratarse de un material diseñado para el desarrollo conjunto de funciones cognitivas y sensomotrices.
\end{abstract}

Palabras clave: Didáctica de la las Ciencias Experimentales | Didáctica de la Matemática | Formación del Profesorado | Juegos Educativos

CIENTIMATES, A PLAYFUL RESOURCE FOR SCIENCE AND MATHEMATICS TEACHING IN KINDERGARTEN EDUCATION

Abstract: With the work summarized in this article, we have conducted the study, design and development of an educational game aimed at kindergarten children, as a tool to help improve understanding of some concepts related to science and mathematics at this stage. Among the authors constituting theoretical background of the developed resource we have Rousseau (1985), in favor of learning by experimentation, Froebel (1902), precursor of the ludic activity for learning, Decroly (1965), who supported the educational value of materials commonly used in everyday life and homemade games, and, especially, Montessori (1937), because this is a resource designed for simultaneous development of cognitive and sensorimotor functions.

Keywords: Science Education | Mathematical Education | Teacher Training | Educational Games

Agradecimientos: Al Secretariado de Innovación Docente del Vicerrectorado de Ordenación Académica y Profesorado de la Universidad de Granada (España), por conceder el Proyecto de Innovación Docente 2013-2016.

\section{Introducción}

Todas las personas independientemente de su raza, edad, sexo y cultura practican el juego en su vida cotidiana de maneras diferentes. Todas las civilizaciones juegan. El juego se vincula más a la infancia pero, en realidad, se presenta a lo largo de toda la vida del hombre. Como bien decía el profesor Cimiano (2003, p. 32), citando a Johan Huizinga, "el juego es más viejo que la cultura; pues, por mucho que estrechemos el concepto de está, presupone siempre una sociedad humana, y los animales no han esperado a que el hombre les enseñe a jugar."

Debido a su importancia, el juego puede ser una muy buena táctica para llegar al aprendizaje y al disfrute de las asignaturas escolares que más problemas, miedos e inseguridades planteen a los alumnos de todas las etapas educativas desde la educación primaria hasta el bachiller. Entre estas, suelen encontrarse las ciencias y las matemáticas. Si hacemos que los niños jueguen con las ciencias y las matemáticas, no solo aprenderán ahora, sino que también podrán seguir haciéndolo a lo largo de su vida. No en vano, los futuros maestros se muestran convencidos de las relaciones existentes entre juego, educación y pensamiento científico y matemático (Fernández-Oliveras y Oliveras, 2014b, 2015). Por ello, decidimos elaborar un juego educativo dirigido a niños de Educación Infantil, como herramienta que ayude a mejorar la comprensión de algunos conceptos relacionados con las ciencias y las matemáticas en esta etapa. 
Entre los autores que constituyen la fundamentación teórica del recurso que hemos elaborado se encuentra Rousseau (1985), partidario del aprendizaje por experimentación, Froebel (1902), precursor de la actividad lúdica para aprender, Decroly (1965), que apoyaba el valor didáctico de materiales de uso común en la vida cotidiana y juegos fabricados en casa, y , especialmente, Montessori (1937), por tratarse de un material diseñado para el desarrollo conjunto de funciones cognitivas y sensomotrices. Las características finales del juego creado se muestran en dos fichas cuyos modelos se desarrollaron en una asignatura correspondiente al último curso del Grado en Educación Infantil de la Universidad de Granada (Fernández-Oliveras y Oliveras, 2014a).

\section{DISEÑO DEL JUEGO}

Se ha considerado que los materiales del juego, fueran reciclados para lograr objetivos educativos y prácticos. Por un lado, para que el coste final fuese lo menor posible, de forma que un maestro pueda abordarlo para realizarlo en su clase, se ha decidido utilizar estos materiales ya que son gratuitos, existe disponibilidad para conseguirlos fácilmente y los alumnos los pueden manipular sin miedo a que se estropeen o se rompan ya que, se pueden sustituir por unos nuevos en cualquier momento. Además, utilizando estos materiales, se muestra a los niños una actitud de cuidado y respeto hacia el medio ambiente.

Se ha querido realizar un juego con un diseño simple pero a la vez muy llamativo, novedoso para los niños. Consta de un tablero que actúa como tapa de la caja que constituye el núcleo del juego, y en cuyo interior se encuentran distintas fichas. En la Figura 1 se presenta un esquema del diseño del recurso.

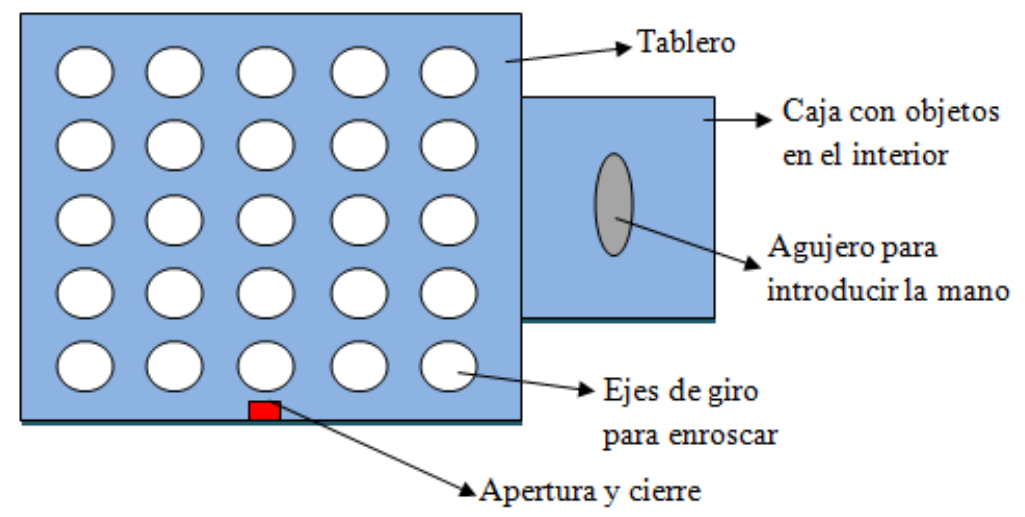

Figura 1. Plano del exterior del juego visto desde arriba.

\section{ELABORACIÓN}

La elaboración del juego es artesanal y sencilla, pero requiere tiempo. Proponemos que se realice implicando a los padres y abuelos de los alumnos que de forma voluntaria quieran colaborar, bien aportando los materiales, o con su trabajo. Seguidamente, se va a explicar la fabricación de cada parte del juego. 
$1^{\circ}$ - Realización del exterior e interior del tablero: La caja grande de cartón se ha reforzado con cinta adhesiva, se ha forrado con papel de ositos (para que quede más llamativa a ojos de los niños) y se han realizado las 6 divisiones que lleva en su interior con trozos de cartón duro de la misma medida que la caja, forrados con papel adhesivo y pegados en su interior con silicona transparente. Dentro de cada división irán las distintas fichas del juego. Finalmente, en la parte superior de la caja, se señala con un rotulador 25 puntos equidistantes donde los tapones-cierres de giro se pegan con silicona transparente.

$2^{\circ}$ - Realización de la segunda caja de juego: La segunda caja que contiene el juego, está realizada con cartón y forrada con el mismo papel. Esta caja no se puede abrir ni cerrar. Sólo, en la parte de arriba se le ha hecho un orificio en forma de ovalo para introducir la mano y los objetos deseados. Dentro de la caja, se han introducido cuatro formas geométricas (triangulo, cuadrado, círculo y rectángulo) y cuatro objetos cotidianos de la vida de los niños como: un lápiz, una llave de metal, una cuchara de plástico y un muñeco pequeño de peluche. Se han elegido estos objetos, además de porque pueden resultar habituales para los niños, porque tienen diferentes cualidades físicas en sus materiales que permiten sean identificados (metal, plástico). Además de estos objetos, se han introducido tiras de papel de periódico dentro de la caja para evitar que los niños puedan ver los objetos que contiene en su interior y hacer que tengan que reconocerlos mediante el tacto. Esta caja, una vez elaborada, va pegada con silicona transparente a la caja con el juego principal.

$3^{\circ}$ - Realización de las fichas de juego: Todas nuestras fichas de juego están elaboradas con tapones de plástico de distintos tamaños. Las fichas de números y signos se han realizado pegando un gomet blanco en la parte de arriba del tapón y escribiendo en él los números del 1 al 25 y 15 signos de suma, resta e igual (5 de cada). Las fichas de letras, se han realizado igualmente del mismo modo, escribiendo las letras en mayúsculas y, debajo, también en minúscula. Las fichas de las distintas tonalidades de colores, se han realizado pintando los gomet de distintos tonos y pegándolos en la parte de arriba del tapón. Las fichas de texturas, se han confeccionado con recortes de tela y papel de distintas texturas (terciopelo, lino, guata, goma-eva, velcro y papel de lija fino) que se han pegado a los tapones con silicona transparente (Figura 2).
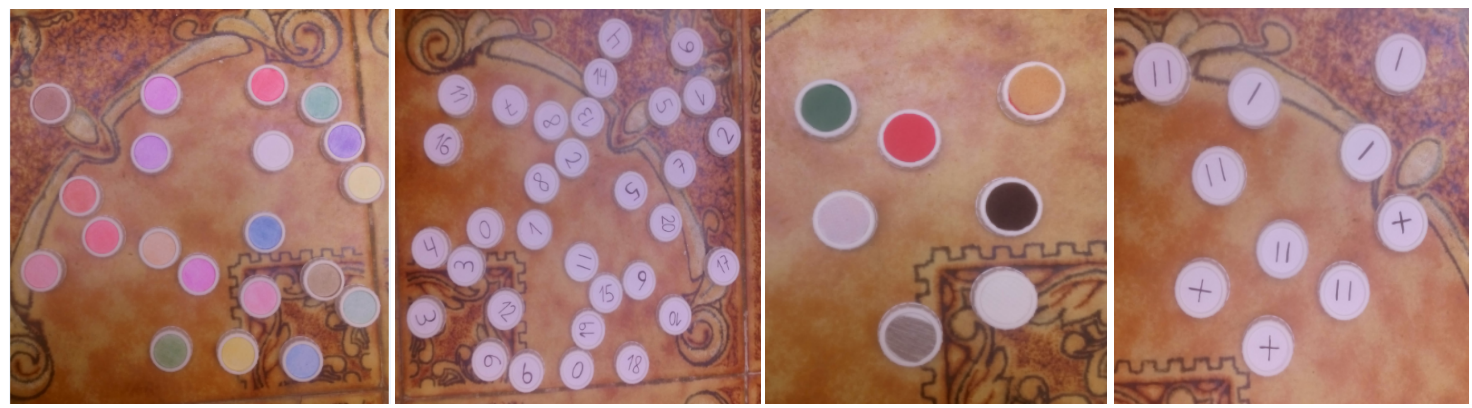

Figura 2. Fichas de, colores, números texturas y signos.

Para la fabricación de las fichas de olores se han seleccionado cinco olores que fueran cotidianos para los niños: pino, flores, limón, menta y canela. Han tenido que emplearse aceites aromáticos artificiales para que los olores sean más intensos y duraderos. Se ha puesto una bolita de algodón en el interior de los tapones y se le ha echado 3 o 4 gotitas de aceite aromático. Después, para cerrar el tapón y evitar que el olor no se evapore con el tiempo, se han introducido los tapones en globos pequeños y se le ha hecho un nudo. Se ha elegido este material para tapar los tapones porque el 
plástico de los globos permite dejar pasar el aroma de las fragancias que hay en su interior y a la vez, evita que se derrame algún liquido que pueda haber en el interior del tapón. De esta manera, las fichas tienen olor, son manipulables e impiden que los niños se manchen.

Las fichas de sabores se han elaborado con tapones medianos. Se han seleccionado 5 condimentos alimenticios en polvo (golosina pica- pica, chocolate puro, azúcar, sal, y pimienta) de distintos sabores básicos (ácido, amargo, dulce, salado y picante, respectivamente), se han echado en el interior de los tapones y se han cerrado con una tapa de cartón duro pegado con silicona transparente. Una vez pegada la tapa, se le han realizado en ella unos agujeritos pequeños para que pueda salir el condimento.

Las fichas de sonidos, se han hecho con tapones grandes en cuyo interior se han metido diferentes objetos (bolitas pequeñas de plástico, tornillos de metal y 40 monedas de un céntimo) y se han cerrado con una tapa de cartón pegada con silicona (Figura 3).
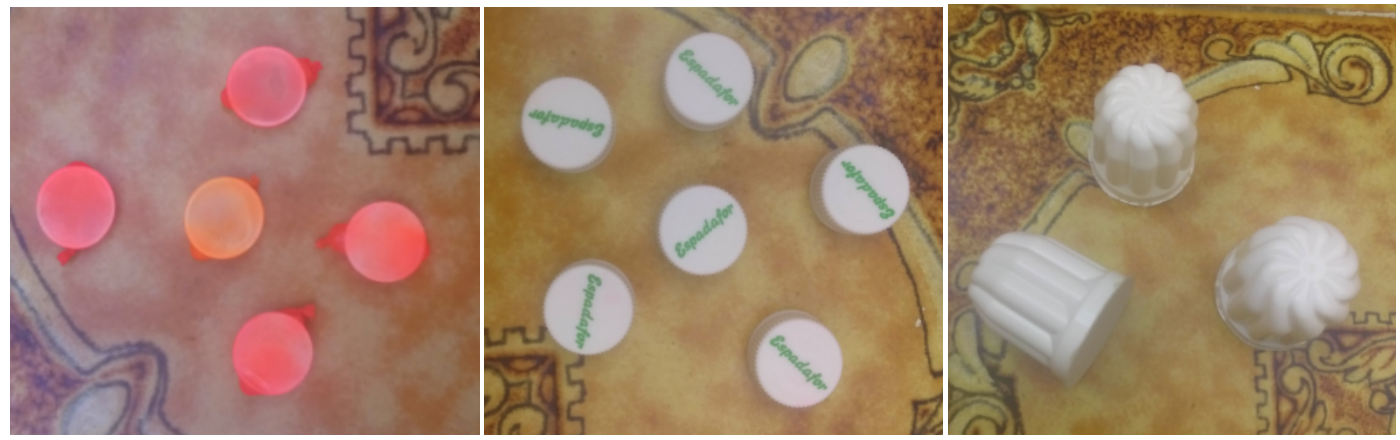

Figura 3. Fichas de olores, sabores y sonidos

\section{FICHAS TÉCNICA Y DIDÁCTICA DEL JUEGO}

Con la idea de que el juego creado pudiera incorporarse al conjunto de recursos disponibles, por ejemplo, en una ludoteca, hemos confeccionado dos fichas que resumen sus características (Tablas 1 y 2 ).

Tabla 1. Ficha técnica del juego Cientimates.

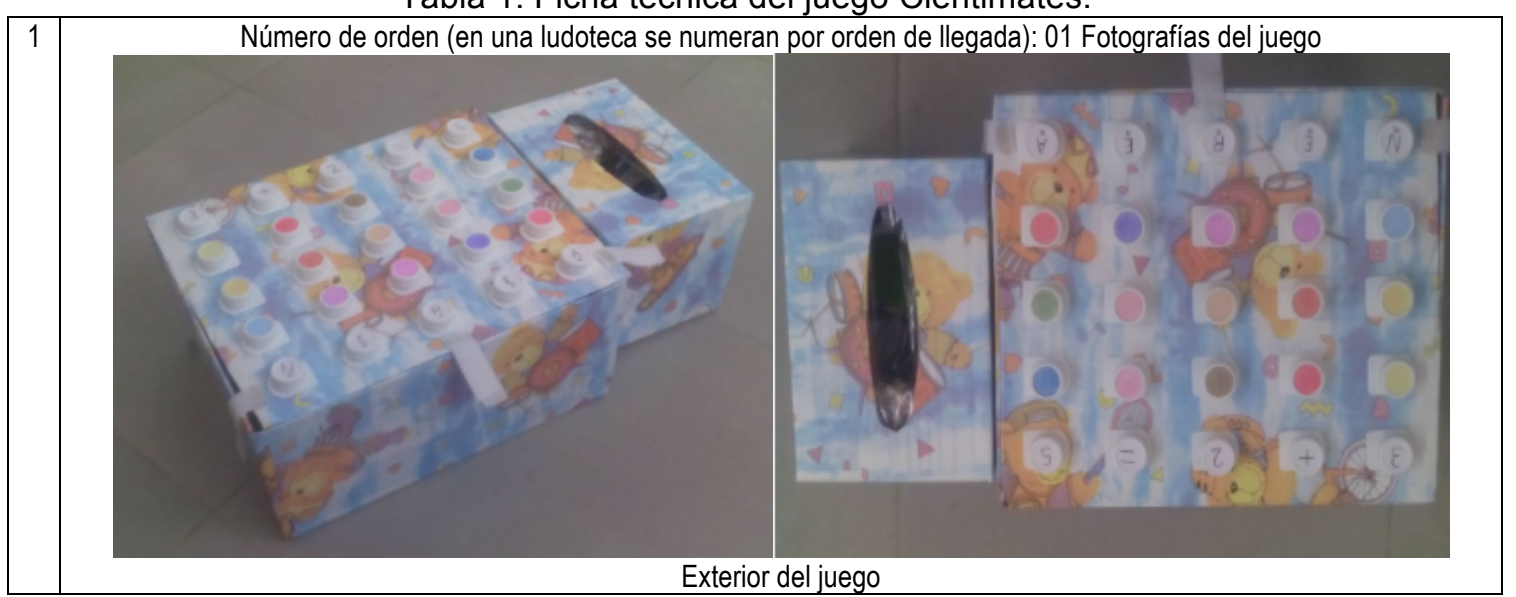




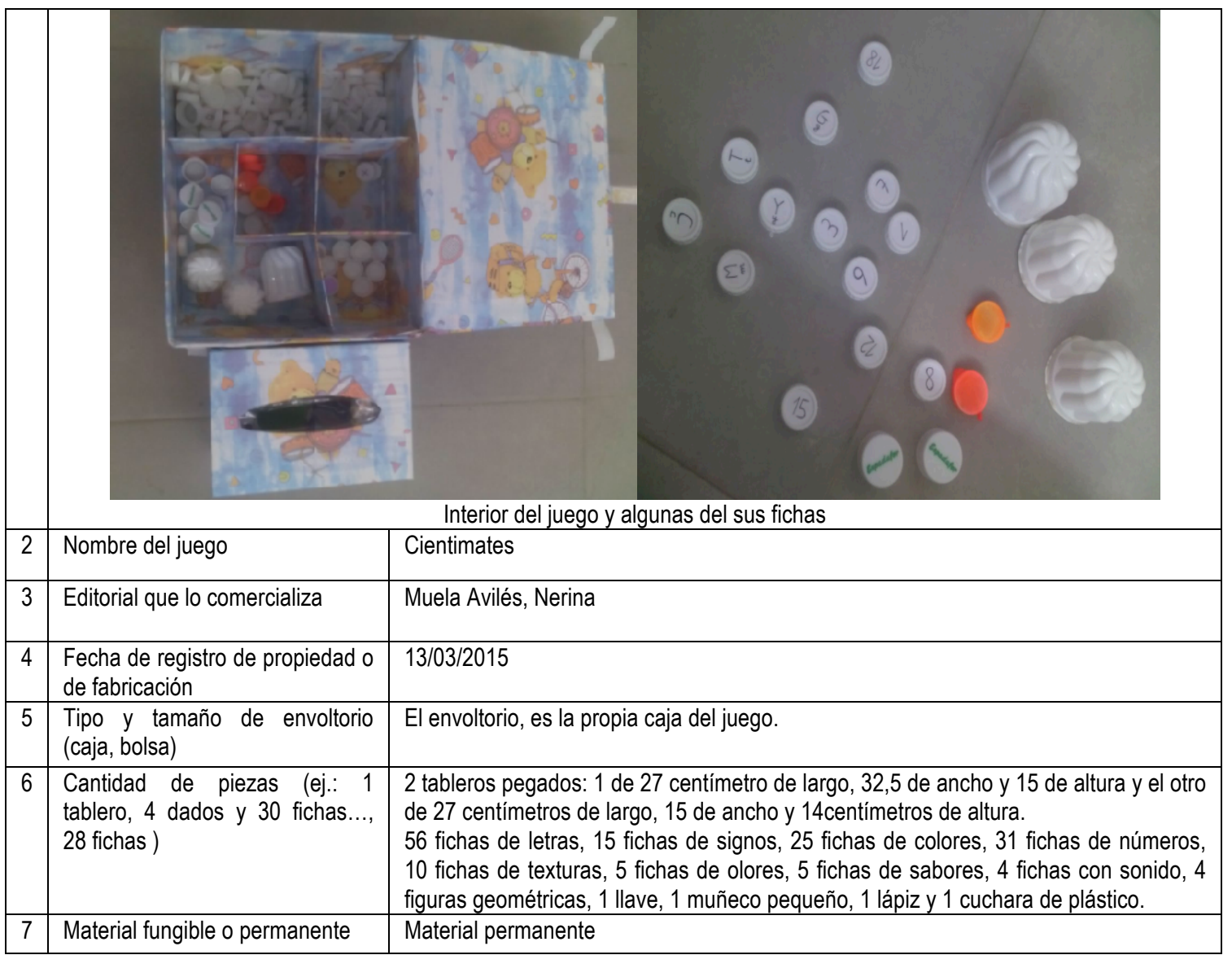

Tabla 2. Ficha didáctica del juego Cientimates.

\begin{tabular}{|c|c|}
\hline Nombre del juego & Cientimates \\
\hline Piezas y material: descripción & $\begin{array}{l}\text { El tablero es rectángulo con medidas de } 27 \text { centímetros de largo, } 32,5 \text { centímetros } \\
\text { de ancho y } 15 \text { centímetros de altura. Realizado con cartón duro y forrado con papel } \\
\text { adhesivo azul con ositos. } \\
\text { Encima del tablero, hay } 25 \text { cierres de plástico, con ejes de giro y de color blanco. } \\
\text { En el interior del tablero, nos encontramos las piezas divididas por compartimentos } \\
\text { realizados también con cartón y forrados. Casi todas las piezas poseen formas } \\
\text { redondas y son de plástico. Podemos encontrar piezas pequeñas, medianas y } \\
\text { grandes. } \\
\text { Al lado del tablero hay una caja con un agujero pequeño para que los niños puedan } \\
\text { introducir la mano. También es de cartón y esta forrada del mismo papel. } \\
\text { Hay un total de } 159 \text { piezas. } \\
56 \text { piezas de letras, } 25 \text { de colores, } 15 \text { de signos, } 31 \text { de números, } 10 \text { de texturas, } 5 \\
\text { piezas con colores, } 4 \text { piezas con sonidos, } 5 \text { piezas con sabores, } 4 \text { figuras } \\
\text { geométricas, y } 4 \text { objetos cotidianos (lápiz, llaves, cuchara de plástico, muñeco) }\end{array}$ \\
\hline Lugar de fabricación & España \\
\hline $\begin{array}{l}\text { Homologación [Sí, en qué país(es): } \\
\text { /No] }\end{array}$ & No \\
\hline $\begin{array}{l}\text { Cualidades intrínsecas de las piezas: } \\
\text { formas y color, material del que están } \\
\text { hechas }\end{array}$ & $\begin{array}{l}\text { Todas las piezas tienen formas redondas, son de plástico duro y poseen un color } \\
\text { blanco excepto } 5 \text { de ellas que son naranjas. }\end{array}$ \\
\hline $\begin{array}{l}\text { Cualidades relativas de las piezas: } \\
\text { tamaños, color, texturas, volumen }\end{array}$ & $\begin{array}{l}\text { En el juego existen piezas de distintos tamaños. Hay piezas pequeñas, medianas y } \\
\text { grandes. }\end{array}$ \\
\hline $\begin{array}{l}\text { Dinámica del juego y reglas: } \\
\text { explicación }\end{array}$ & $\begin{array}{l}\text { Se juega de uno en uno y tienen que ir realizando todas las pruebas del tablero. Las } \\
\text { pruebas son las siguientes: } \\
\text { Primero la prueba de números donde tienen que realizar una suma o resta sencillas. } \\
\text { La segunda prueba es de colores. Los niños tienen que identificar el color y su } \\
\text { tonalidad de dos fichas. } \\
\text { La tercera prueba es la de letras, donde los niños tienen que escribir su nombre. }\end{array}$ \\
\hline
\end{tabular}




\begin{tabular}{|c|c|}
\hline & $\begin{array}{l}\text { La cuarta prueba es la de texturas. Los niños tienen que identificar con los ojos } \\
\text { cerrados la textura de la ficha. } \\
\text { La quinta prueba es de olores. Los participantes tienen que oler dos fichas y } \\
\text { reconocer su aroma. } \\
\text { La sexta prueba es de sabores. Los niños tienen que identificar el olor de dos fichas } \\
\text { del juego. } \\
\text { La séptima prueba es de sonidos. Los niños tienen que las dos fichas que tienen el } \\
\text { mismo sonido. } \\
\text { La última prueba es de tacto, donde, se tiene que introducir la mano en el agujero, } \\
\text { tocar dos objetos e identificarlos. }\end{array}$ \\
\hline $\begin{array}{l}\text { Peligrosidad/riesgos (explicar si en las } \\
\text { piezas o en su uso con determinadas } \\
\text { reglas) }\end{array}$ & No existe ningún peligro en la realización del juego. \\
\hline Tiempo de preparación & No precisa de tiempo de preparación. \\
\hline Duración [corta/media/larga] & Se precisa una duración media \\
\hline $\mathrm{N}^{0}$ de jugadores & De 1 a 2 jugadores \\
\hline Edad preferente de los jugadores & De 3 a 6 años \\
\hline De uso en interior/exterior & Se puede jugar tanto en interior como en exterior. \\
\hline $\begin{array}{l}\text { Tipos de acciones de los jugadores } \\
\text { primordialmente (físicas, mentales, } \\
\text { ambas) }\end{array}$ & $\begin{array}{l}\text { Los jugadores mediante el juego, realizan ambas acciones, tanto físicas como } \\
\text { mentales. }\end{array}$ \\
\hline Sentidos que se utilizan & En este juego, se fomentan los 5 sentidos (olfato, gusto, tacto, vista y oído) \\
\hline $\begin{array}{l}\text { Habilidades/capacidades o destrezas } \\
\text { que favorece (psicomotrices, } \\
\text { cognitivas...) }\end{array}$ & $\begin{array}{l}\text { El juego fomenta algunas capacidades cognitivas como son por ejemplo: la } \\
\text { memorización, el pensamiento, el razonamiento y la atención. Favorece las } \\
\text { capacidades sensoriales del gusto, el tacto, la vista, el olfato y el oído y favorece la } \\
\text { destreza óculo-manual de enroscar y desenroscar tapones }\end{array}$ \\
\hline Objetivos propios del juego & $\begin{array}{l}\text {-Aprender o repasar la suma. } \\
\text {-Aprender o repasar la resta. } \\
\text {-Aprender palabras. } \\
\text {-Aprender o repasar los colores. } \\
\text {-Reconocer algunos olores (limón, canela, menta, pino y rosa). } \\
\text {-Reconocer los sabores probando diferentes alimentos (sal, azúcar, chocolate } \\
\text { amargo, pica-pica y pimienta). } \\
\text {-Reconocer texturas mediante el tacto (rugoso, suave, duro, blanco...). } \\
\text {-Reconocer sonidos a través de distintos botes. } \\
\text {-Reconocer o aprender algunas figuras geométricas (circulo, cuadrado, rectángulo y } \\
\text { triangulo). } \\
\text {-Reconocer algunos objetos cotidianos por sus propiedades físicas (llaves de metal, } \\
\text { cuchara de plástico, muñeco pequeño suave, lápiz duro...). }\end{array}$ \\
\hline $\begin{array}{l}\text { Coste económico (estimado o } \\
\text { conocido) }\end{array}$ & 5 euros. \\
\hline Contenidos matemáticos & $\begin{array}{l}\text { La suma, la resta, los signos, los números y las figuras geométricas (círculo, } \\
\text { cuadrado, rectángulo y triangulo) }\end{array}$ \\
\hline Contenidos científicos & $\begin{array}{l}\text { Los cinco sentidos (olfato, gusto, oído, vista y tacto) y algunos conceptos } \\
\text { relacionados con las propiedades de la materia como el color, dureza, fragilidad... }\end{array}$ \\
\hline Autocorrección [Sí/No] & No posee autocorrección \\
\hline $\begin{array}{l}\text { Puede ser comprendido sin } \\
\text { explicaciones [Si/No] }\end{array}$ & No puede ser comprendido sin explicaciones a edades tan pequeñas. \\
\hline $\begin{array}{l}\text { Fundamentación teórica (autores en } \\
\text { los que se basa) }\end{array}$ & Rousseau (1985), Montessori (1937), Fröebel (1902), Decroly (1965). \\
\hline
\end{tabular}

\section{Reflexión final}

Con el trabajo resumido en este artículo, hemos podido comprobar el proceso que lleva consigo la elaboración de un juego educativo científico y matemático para Educación Infantil, desde la concepción de la idea inicial hasta la realización casera de su fabricación.

\section{Referencias}

Cimiano, J. G. (2003). El homo ludens de Johan Huizinga. Retos: nuevas tendencias en educación física, deporte y recreación, (4), 32-35. 
Decroly O. (1965). La Fonction de Globalisation et l'Enseignement. Brussels: Editions Desoer.

Fernández-Oliveras, A. y Oliveras, M. L. (2014a). Playing for science and mathematics education: an experience for pre-service kindergarten teacher training. En Costa M. F. M., Pombo P., Dorrío B.V. (Eds.), Hands-on Science. Science Education with and for Society (180 -183). Braga: Hands-on Science Network.

Fernández-Oliveras, A. y Oliveras, M. L. (2014b). Pre-service kindergarten teachers' conceptions of play, science, mathematics, and education. Procedia - Social and Behavioral Sciences, 152, 856-861. DOI:10.1016/j.sbspro.2014.09.334.

Fernández-Oliveras, A. y Oliveras, M. L. (2015). Conceptions of science, mathematics, and education of prospective kindergarten teachers in a play-based training. International Journal on Advances in Education Research, 2(1), 37-48.

Froebel, F. (1902). La educación del hombre. Nueva York: D. Appleton y Compañía.

Montessori, M. (1937). El método de la pedagogía científica aplicado a la educación. Barcelona: Araluce.

Real Decreto por el que se establecen las enseñanzas mínimas del segundo ciclo de Educación infantil. (Real Decreto 1630/2006, 29 de diciembre). Boletín Oficial del Estado, $n^{\circ} 4$, 2007, 4 enero.

Rousseau, J. J. (1985). Emilio o de la Educación. Madrid: Edaf. 